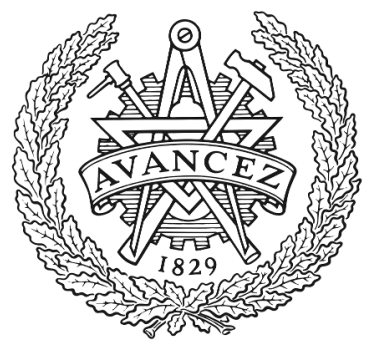

CHALMERS

UNIVERSITY OF TECHNOLOGY

\title{
Vector-corrected Nonlinear Multi-port IQ-mixer Characterization using Modulated Signals
}

Downloaded from: https://research.chalmers.se, 2023-04-26 14:56 UTC

Citation for the original published paper (version of record):

Gustafsson, S., Thorsell, M., Buisman, K. et al (2017). Vector-corrected Nonlinear Multi-port IQ-mixer Characterization using Modulated Signals. IEEE MTT-S International Microwave Symposium Digest: 1433-1436. http://dx.doi.org/10.1109/MWSYM.2017.8058888

N.B. When citing this work, cite the original published paper. 


\title{
Vector-corrected Nonlinear Multi-port IQ-mixer Characterization using Modulated Signals
}

\author{
Sebastian Gustafsson, Mattias Thorsell, Koen Buisman and Christian Fager \\ Chalmers University of Technology, SE-412 96 Gothenburg, Sweden
}

\begin{abstract}
In this paper, large-signal operation of IQ-mixers is studied using a vector-corrected four-port measurement setup with modulated signals as stimuli. The measurement setup presents unique characterization possibilities since it has two ports at low/baseband frequencies and two ports at RF, making it ideal for characterization of frequency-translating devices such as mixers. A commercial upconverting IQ-mixer is studied, with the $I$ and $Q$ input signals residing at incommensurate frequency grids, enabling separation of the nonlinear distortion generated in the $I$ and $Q$ branches. Frequency-domain and time-domain measurements reveal imbalances between the $I$ and $Q$ branches in terms of conversion gain and nonlinear distortion. It is also shown for the same mixer that operating the $I$ and $Q$ branches concurrently has limited influence on both conversion gain and nonlinear distortion, compared to non-concurrent operation.

Index Terms-Microwave measurement, Nonlinear distortion, Mixers, Frequency-domain analysis, Time-domain analysis.
\end{abstract}

\section{INTRODUCTION}

To increase the capacity in future wireless communication networks, wideband communication signals at higher carrier frequencies are necessary. Despite recent advances in highspeed digital-to-analog converters, frequency-translating devices such as mixers are still vital components in microwave systems.

The upconverting IQ-mixer has two inputs at baseband, lowfrequency (LF), labeled in-phase (I) and quadrature-phase (Q). The orthogonal combination of these two inputs is upconverted to RF using the multiplicative properties of e.g. a diode driven by a local oscillator (LO). Ideally, this procedure results in a linear translation of the signal content at LF up to RF. In practice however, there is not only in-band and out-of-band nonlinear distortion but also conversion imbalances between the I and Q branches. Since the mixer is an integral part of a microwave system, it is important to both characterize and model it properly to enable compensation of its nonidealities.

Previous work on the topic of microwave mixer characterization includes nonlinear $\mathrm{CW}$ measurements for extraction of various transfer functions [1], formulation of scatteringparameter models for mixers [2] and vector mixer characterization [3]. Although these papers provide useful insight on general mixer behavior, they do not cover four-port characterization of IQ-mixers using modulated signals.

In this paper, a vector-corrected nonlinear measurement setup capable of characterizing frequency-translating devices is used to study nonlinear distortion and I and Q branch imbalances in a commercially available IQ-mixer. The nonlinearities and branch imbalances are assumed to occur at baseband, whilst the upconversion is a linear frequency-translation from

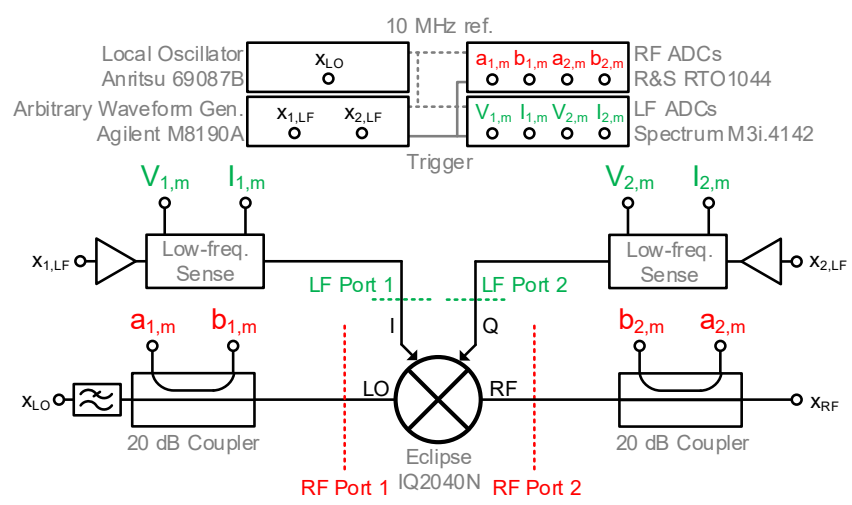

Fig. 1. Block diagram of the four-port measurement setup.

LF to RF. The baseband input signals consist of multisines with specially designed frequency grids, which enables separation of the upconverted RF spectrum into linear and nonlinear contributions from the I and Q branches.

\section{Measurement Setup Description}

The measurement setup used in this paper builds upon the work of [4]. Rather than measuring LF and RF at the same reference planes, the LF and RF measurement ports are in this paper separated, as shown in Fig. 1. Hereby, four fully calibrated measurement ports are available, two at low frequency (DC-125 MHz) and two at high frequency (300 MHz-4 GHz). Frequency-translating devices can thus readily be measured. All of the instruments in the setup share a $10 \mathrm{MHz}$ reference clock, and the arbitrary waveform generator triggers the LF and RF digitizers. The calibration routine of the setup, which includes absolute power and phase calibration, is based on the methods presented in [4], [5].

\section{A. Signal Generation}

The periodic excitation signals used for characterization are based on random phase multisines. More specifically, odd random phase multisines with random harmonic grid are used [6]. By using an odd frequency excitation grid, distortion due to odd nonlinearities will end up on odd frequency bins and distortion due to even nonlinearities will end up on even frequency bins. Furthermore, by leaving certain excitation frequencies empty, random detection lines are introduced, allowing for separation and determination of both odd and even nonlinearities. 
Measured RF spectrum
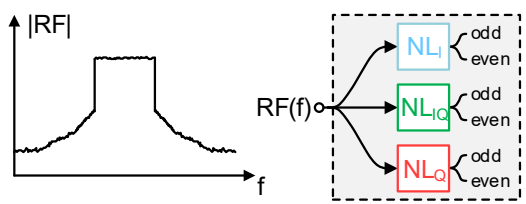

Decomposed RF spectrum

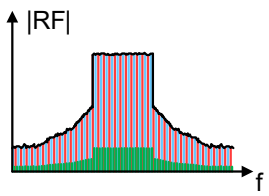

Fig. 2. Decomposition of the measured IQ-mixer RF output signal, $R F(f)$, into contributions from the I branch $\left(\mathrm{NL}_{\mathrm{I}}\right), \mathrm{Q}$ branch $\left(\mathrm{NL}_{\mathrm{Q}}\right)$ and inter-branch interaction $\left(\mathrm{NL}_{\mathrm{IQ}}\right)$.

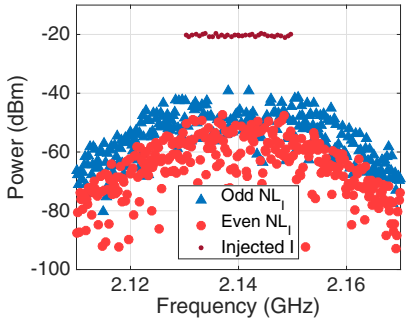

(a)

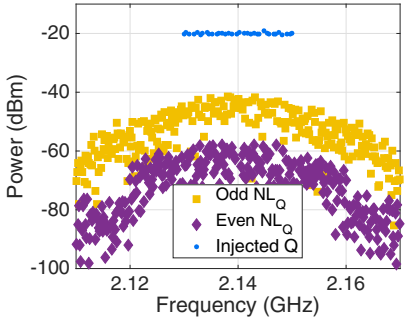

(b)
Fig. 3. RF output spectra with separation of odd and even nonlinear distortion for non-concurrent IQ operation with high LF drive power. (a) RF spectrum when exciting the I branch. (b) RF spectrum when exciting the Q branch.

For the measurements presented in this paper, the upconverting IQ-mixer can be considered as a multiple-input singleoutput system, with two LF inputs (I and Q branches), one $\mathrm{RF}$ input (LO branch) and one RF output (RF branch). In conventional usage, the I and $\mathrm{Q}$ branches are concurrently excited with overlapping excitation frequencies. Hence, distortion due to nonlinearities in the I and Q branches will end up on the same frequency grid at RF. It is therefore hard, if not impossible, to separate distortion generated in either of the two branches and imbalances between these.

In this paper, the excitation frequency grid for $\mathrm{I}$ and $\mathrm{Q}$ is differentiated such that the upconverted RF spectrum and the nonlinearities can be separated into contributions from the I and Q branch respectively, as well as intermodulation products between the two branches, as shown in Fig. 2. In combination with odd random phase multisines with random harmonic grid, the odd and even nonlinear distortion can be separated as well.

In the measurements presented in this paper, the excitation frequency grid has a frequency spacing of $\Delta f_{I}=99 \mathrm{kHz}$ for the I branch and $\Delta f_{Q}=101 \mathrm{kHz}$ for the Q branch. The excitation frequency grids are thus defined as

$$
\begin{aligned}
f_{I, k} & =\Delta f_{I} \cdot k, \quad k=1,3, \ldots\left\lfloor f_{B W} / \Delta f_{I}\right\rfloor, \\
f_{Q, k} & =\Delta f_{Q} \cdot k, \quad k=1,3, \ldots\left\lfloor f_{B W} / \Delta f_{Q}\right\rfloor,
\end{aligned}
$$

where $f_{I, k}$ and $f_{Q, k}$ are the excitation frequency grids for I and $\mathrm{Q}$ respectively, and $f_{B W}$ is the excitation bandwidth. Some frequencies from these excitation grids have however been randomly removed in order to serve as detection lines for odd nonlinearities [6].

Nonlinear distortion residing at common multiple frequencies of the I and Q frequency grid can not be separated. Here,

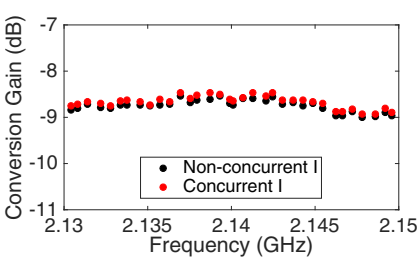

(a)

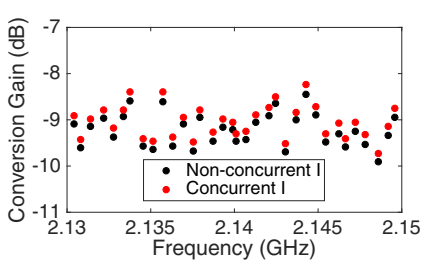

(c)

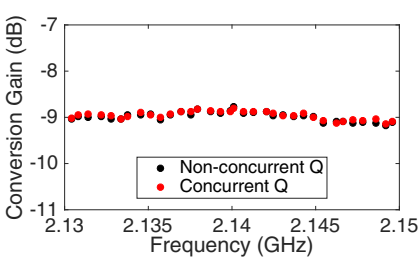

(b)

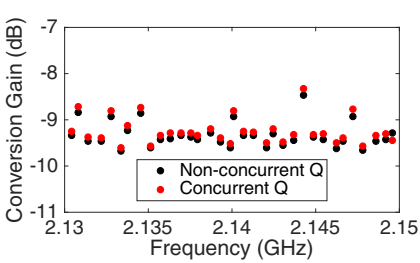

(d)
Fig. 4. Conversion gain measured at two different LF drive power levels for non-concurrent IQ operation and concurrent IQ operation. (a) Conversion gain for the I branch at low drive power. (b) Conversion gain for the Q branch at low drive power. (c) Conversion gain for the I branch at high drive power. (d) Conversion gain for the $\mathrm{Q}$ branch at high drive power.

the lowest common multiple frequency is $9.99 \mathrm{MHz}$, which can be increased by e.g. reducing the frequency grid spacing, resulting in longer measurement times.

\section{RESUlTS}

The measurements presented in this section were carried out on an IQ-mixer from Eclipse Microwave (IQ2040N). The mixer has an IF range of $\mathrm{DC}-500 \mathrm{MHz}$ and an $\mathrm{RF}$ range of $2 \mathrm{GHz}-4 \mathrm{GHz}$. The LO frequency was set to $2.14 \mathrm{GHz}$ and the LO drive power was $13 \mathrm{dBm}$. The load impedance on the mixer RF port was set to roughly $50 \Omega$ by passively terminating the $\mathrm{x}_{\mathrm{RF}}$ port in Fig. 1.

The mixer was characterized under two different modes of operation. First, the I and Q branches were excited individually, here denoted as non-concurrent IQ characterization. The I and Q branches were then excited simultaneously, here denoted as concurrent IQ characterization. In both modes of operation, a random-phase multisine signal with 17 tones and $10 \mathrm{MHz}$ excitation bandwidth was injected on the I and Q branch, respectively. The phases of the tones were randomized such that the peak-to-average power ratio (PAPR) was $10 \mathrm{~dB}$.

The IQ-mixer was characterized at two different LF drive powers, denoted low drive power $(-7.6 \mathrm{dBm}$ average power) and high drive power (1.4 $\mathrm{dBm}$ average power). The measurement noise floor has been excluded from the RF spectrum plots for readability, and is roughly $-80 \mathrm{dBm}$.

\section{A. Non-concurrent IQ Characterization}

The RF output spectra for the high LF drive power is shown in Fig. 3. The odd nonlinear distortion for the two branches are at roughly the same level, $-20 \mathrm{dBc}$, while the even nonlinear distortion in the I branch are roughly $10 \mathrm{~dB}$ higher than the even nonlinear distortion in the Q branch. This is a significant difference which needs to be accounted for in e.g. IQ imbalance compensation algorithms. 


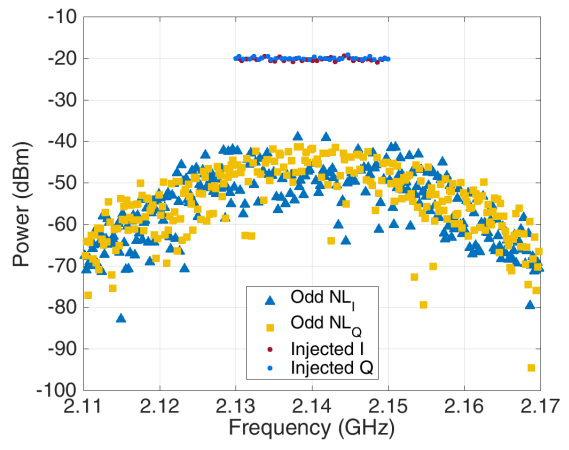

(a)

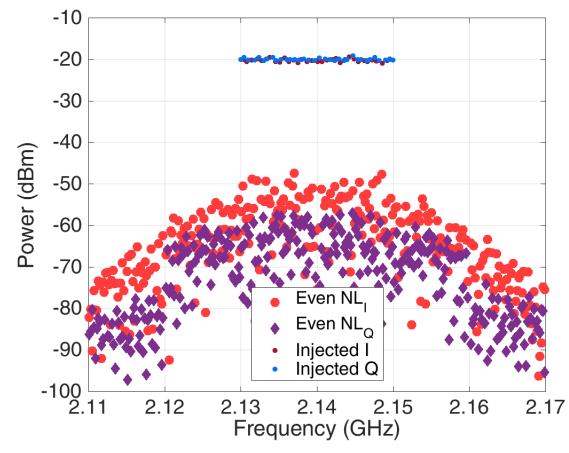

(b)

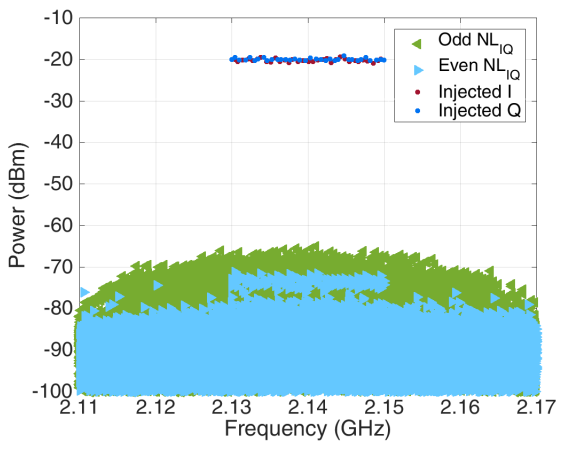

(c)

Fig. 5. RF output spectra with separation of odd and even nonlinear distortion for concurrent IQ operation with high LF drive power. (a) Odd nonlinear distortion. (b) Even nonlinear distortion. (c) Odd and even inter-branch nonlinear distortion.

\section{B. Concurrent IQ Characterization}

The conversion gain for the low and high LF drive power under non-concurrent and concurrent IQ operation is shown in Fig. 4. The imbalance between the I and Q branches under low drive power, Fig. 4a and Fig. $4 \mathrm{~b}$, is roughly $0.2 \mathrm{~dB}$ in average and the conversion gain is little affected by concurrent operation. For higher drive powers, Fig. $4 \mathrm{c}$ and Fig. 4d, the imbalance is in average slightly less, but there is some gain expansion in the two branches under concurrent operation. The gain expansion is approximately $0.2 \mathrm{~dB}$ for the I branch and $0.1 \mathrm{~dB}$ for the $\mathrm{Q}$ branch, and is relatively consistent across the frequency band.

The RF output spectra for the high LF drive power is shown in Fig. 5. The measured odd nonlinear distortion, Fig. 5a, are consistent with the results from the non-concurrent characterization. The measured even nonlinear distortion, Fig. 5b, also show similar results compared to the non-concurrent characterization, with about $10 \mathrm{~dB}$ higher levels in the I branch relative to the Q branch. In Fig. 5c, intermodulation products between the I and Q frequencies are shown. Since the I and Q branches are excited at non-overlapping frequency grids, this figure shows any nonlinear interaction between the branches. There is some difference between odd and even nonlinear distortion, however the levels are relatively low at this LF drive power and has therefore a small impact on the total output signal.

\section{Time-domain Characterization}

The LF and RF time-domain waveforms were reconstructed from an inverse Fourier transform of the measured and calibrated frequency spectra. Since the calibration routines of the measurement setup include phase calibration, the relative phases between the tones are preserved and the obtained timedomain waveforms are thus an accurate representation of the true waveforms. Time-alignment of the LF and RF envelope waveforms was carried out to eliminate the effects of trigger jitter, which was in the order of 5 ns.

The time-domain measurement results at high LF drive power are shown in Fig. 6. Minor differences in the amplitude distortion for the I and Q branches, Fig. 6a and Fig. 6b,

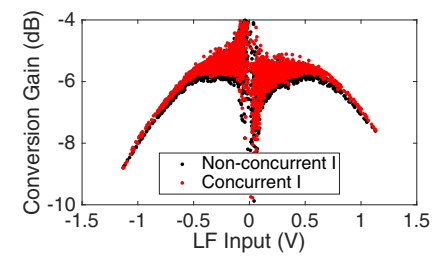

(a)

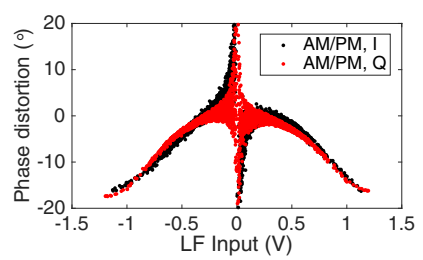

(c)

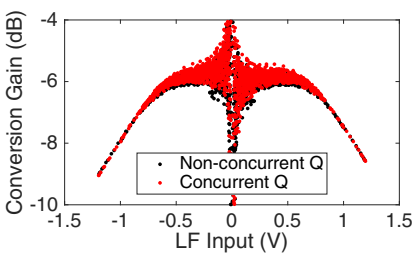

(b)

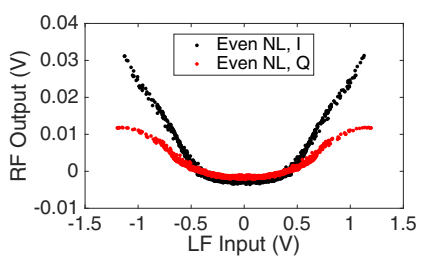

(d)
Fig. 6. Time-domain characterization of the IQ-mixer. (a) I RF amplitude distortion versus I LF input voltage. (b) Q RF amplitude distortion versus Q LF input voltage. (c) RF phase distortion versus LF input voltage under concurrent operation. (d) RF output voltage of the even nonlinear distortion versus LF input voltage under concurrent operation.

are seen, although the compression characteristics are slightly different. Furthermore, the difference between non-concurrent and concurrent operation is very subtle in the AM/AM plots. Phase distortion in the two branches under concurrent operation, Fig. 6c, is comparable with roughly $17^{\circ}$ distortion at peak power. The time-domain signal of the even nonlinear distortion under concurrent operation is shown in Fig. 6d. For the Q branch, the even nonlinear distortion seems to compress at higher drive powers, whereas for the I branch they continue to increase in amplitude for higher drive powers, thus explaining the increased levels of even nonlinear distortion in the I branch, as seen in Fig. 5b.

\section{CONCLUSION}

A vector-corrected measurement setup capable of simultaneous LF and RF measurements of frequency-translating devices has been used for the characterization of a commercially available IQ-mixer. The measurement capabilities provided by 
the setup gives unique insight into the large-signal operation of IQ-mixers. Furthermore, the utilization of a specially designed frequency grid enables separation of odd and even nonlinearities as well as separation of nonlinear contributions from the I and Q branches under concurrent operation. Measurements reveal imbalances in the two branches in terms of conversion gain and nonlinear distortion.

\section{ACKNOWLEDGMENT}

The results in this paper are partly the result of the project MET5G Metrology for 5G communications. This project has received funding from the EMPIR programme co-financed by the Participating States and from the European Unions Horizon 2020 research and innovation programme. Furthermore this research has been carried out in the GigaHertz centre in a joint research project financed by Swedish Governmental Agency of Innovation Systems (VINNOVA), Chalmers University of Technology, SweGaN AB, Comheat Microwave AB, Ericsson AB, Infineon Technologies Austria AG, Mitsubishi Electric Corporation, Saab AB, SP Technical Research Institute of Sweden, and United Monolithic Semiconductors.

\section{REFERENCES}

[1] W. Van Moer and Y. Rolain, "Proving the usefulness of a 3-port nonlinear vectorial network analyser through mixer measurements," in 2003 IEEE MTT-S Int. Microw. Symp. Dig., Philadelphia, PA, USA, Jun. 2003.

[2] D.F. Williams, F. Ndagijimana, K.A. Remley, J.A. Dunsmore and S Hubert, "Scattering-parameter models and representations for microwave mixers," IEEE Trans. Microw. Theory Techn, vol. 53, iss. 1, pp. 314-321, Jan. 2005.

[3] J.A. Dunsmore, "Novel method for vector mixer characterization and mixer test system vector error correction," in 2002 IEEE MTT-S Int. Microw. Symp. Dig., Seattle, WA, USA, Jun. 2002.

[4] S. Gustafsson, C. Fager, K. Buisman and M. Thorsell, "Wideband RF characterization setup with high dynamic range low frequency measurement capabilities," in 2016 87th ARFTG Microw. Meas. Conf., San Francisco, CA, USA, Jun. 2016.

[5] S. Gustafsson, M. Thorsell, J. Stenarson and C. Fager, "An Oscilloscope Correction Method for Vector-Corrected RF Measurements," IEEE Trans. Instrum. Meas., vol. 64, iss. 9, pp. 2541-2547, Mar. 2015.

[6] R. Pintelon and J. Schoukens, "Detection, Quantification, and Qualification of Nonlinear Distortions in FRF Measurements," in System Identification - A Frequency Domain Approach, 2nd ed. Hoboken, USA: John Wiley \& Sons, 2012, ch. 4, sec. 4.2, pp. 120-122. 